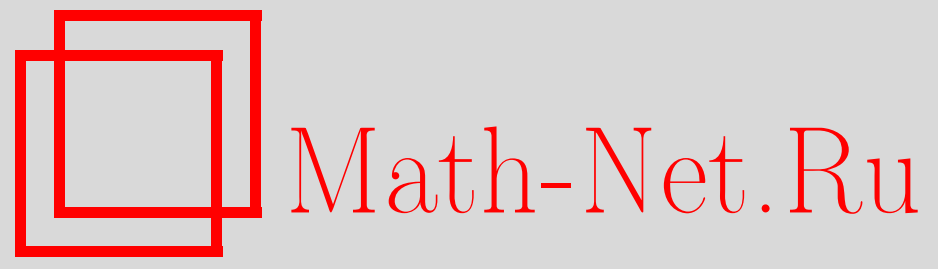

А. Я. Кузнецова, О смесях одновершинных распределений, Теория вероятн. и ее примен., 2006, том 51, выпуск 3, 607-608

DOI: https://doi.org/10.4213/tvp43

Использование Общероссийского математического портала Math-Net.Ru подразумевает, что вы прочитали и согласны с пользовательским соглашением

http: //www.mathnet.ru/rus/agreement

Параметры загрузки:

IP : 54.198 .55 .26

26 апреля 2023 г., 12:12:17

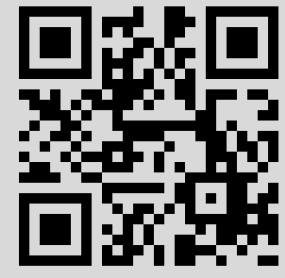


14. Rosenblatt $M$. A central limit theorem and a strong mixing condition. - Proc. Natl. Acad. Sci. USA, 1956 , v. 42 , p. 43-47.

15. Веретенников А. Ю. Об оценках скорости перемешивания для стохастических уравнений. - Теория вероятн. и ее примен., 1987, т. 32, в. 2, с. 299-308.

16. Веретенников $А$. Ю. О полиномиальном перемешивании и скорости сходимости для стохастических дифференциальных и разностных уравнений. - Теория вероятн. и ее примен., 1999, т. 44 , в. 2, с. 312-327.

Поступила в редакцию

14.X.2002

Исправленный вариант

23.V.2005

(C) $2006 r$.

КУЗНЕЦОВА А. Я.*

\section{О СМЕСЯХ ОДНОВЕРШИННЫХ РАСПРЕДЕЛЕНИЙ}

В работе отмечаются некоторые полезные свойства смесей одновершинных распределений.

Ключевые слова и фразы: одновершинные распределения, смеси распределений, отклонение распределений от равномерного.

Мы ограничимся здесь специальным подклассом класса одновершинных распределений.

По определению, плотность вероятности $p(x ; X)$ случайной величины $X$ принадлежит классу CU (Continuous Unimodal), если она непрерывна и ограничена и существует такое $a$, что она не убывает при $x<a$ и не возрастает при $x>a$.

Имеет место следующее утверждение.

Лемма 1. Пусть $U-$ какая-либо случайная величина, плотность вероятности $p(x ; U)$ которой входит в класс CU. Тогда плотность вероятности $p(x ;\{U\})$ ее дробной части $\{U\}$ удовлетворяет неравенству: при любом $0 \leqslant x \leqslant 1$

$$
|p(x ;\{U\})-1| \leqslant 2 \max _{x} p(x ; U)
$$

(см. $[1$, пример $5, \S 8$, гл. II]).

Следствие 1. Пусть $\eta>0$ - масштабный параметр. Тогда в условиях леммы 1

$$
|p(x ;\{\eta U\})-1| \leqslant \frac{2}{\eta} \max p(x ; U) .
$$

Типичные примеры применения следствия 1 см. в [2] и [3].

Перейдем теперь к рассмотрению смесей (см. $[1, \S 5$, гл. II]) плотностей класса CU.

Пусть $U_{1}, \ldots, U_{k}$ - какие-либо случайные величины, плотность которых входит в класс $\mathrm{CU}$, и $p(x ; U)=\sum_{j=1}^{k} p\left(x ; U_{j}\right) \lambda_{j}$, где $\lambda_{j} \geqslant 0, \sum_{j=1}^{k} \lambda_{j}=1$.

Из следствия 1 с очевидностью вытекает

Следствие 2. При всех $0 \leqslant x \leqslant 1$

$$
|p(x ;\{\eta U\})-1| \leqslant \frac{2}{\eta} \sum_{j=1}^{k} \lambda_{j} \max p\left(x ; U_{j}\right) .
$$

* Московский государственный университет им. М.В. Ломоносова, факультет вычислительной математики и кибернетики, Ленинские горы, 119991 Москва, Россия. 
П р и м е р. Допустим, что $U_{j}$ имеет нормальное распределение с параметрами $a_{j}$ и $\sigma_{j}^{2}$. Тогда правая часть неравенства в следствии 2 не превосходит величины $(2 / \eta)\left[\left(\min _{j} \sigma_{j}\right) \sigma \sqrt{2 \pi}\right]^{-1}$.

Лемма 2. Пусть $Y_{1}, \ldots, Y_{n}$ - независимые случайные величинь, причем $Y_{j}$ распределена равномерно на интервале $\left(a_{j}, b_{j}\right), j=1, \ldots, n$.

Тогда сумма $Y_{1}+\cdots+Y_{n}$ имеет одновершинное распределение.

Доказательство следует из результата А. Винтнера (А. Wintner), цитированного в [4, с. 256], см. также [1, задачи 24 и $26, \S 12$, гл. V].

Теорема 1. Пусть $X_{1}, \ldots, X_{n}$ - независимые случайные величины и плотность вероятности каждой из них входит в класс CU. Тогда плотность вероятности их суммы $S=X_{1}+\cdots+X_{n}$ является пределом конечных смесей одновершинных плотностей класса $\mathrm{CU}$.

В доказательстве можно применить рассуждения, использованные в доказательстве теоремы Л. Шеппа $[1$, гл. V, между формулами (9.7) и (9.8)].

\section{СПИСОК ЛИТЕРАТУРЫ}

1. Феллер В. Введение в теорию вероятностей и ее приложения. Т. 2. М.: Мир, 1984, $752 \mathrm{c}$.

2. Куликова А.А., Прохоров Ю.В. Односторонние устойчивые распределения и закон Бенфорда. - Теория вероятн. и ее примен., 2004, т. 49, в. 1, с. 178-184.

3. Куликова А. А., Прохоров Ю. В., Хохлов В. И. Н.F.D. (H-Function Distribution) и закон Бенфорда. - Теория вероятн. и ее примен., 2005, т. 50, в. 2, с. 366-372.

4. Gnedenko B. V., Kolmogorov A.N. Limit Distributions for Sums of Independent Random Variables. Reading, MA: Addison-Wesley, 1954.

Поступила в редакцию

15.XI.2005

(c) 2006 г.

РАДЧЕНКО В. Н. ${ }^{*}$

\section{ХЕДЖИРОВАНИЕ НАИМЕНЬШЕЙ ВАРИАЦИИ В МОДЕЛИ СО СКАЧКАМИ В НЕСЛУЧАЙНЫЕ МОМЕНТЫ ВРЕМЕНИ ${ }^{1)}$}

Рассматривается модель, в которой цена акции управляется винеровским процессом и дополнительно имеет случайные изменения в некоторые заранее известные неслучайные моменты времени. Выводится в явном виде стратегия хеджирования наименьшей вариации для Европейского опциона колл. Вывод основан на разложении Фёльмера-Швайцера функции платежа.

Ключевые слова и фразы: хеджирование наименьшей вариации, Европейский опцион колл, разложение Фёльмера-Швайцера, модель цены акции со скачками, неслучайные моменты скачков, минимальная мартингальная мера.

* Киевский национальный университет им. Тараса Шевченко, кафедра математического анализа, ул. Владимирская, 60, 01033 Киев, Украина; е-mail: vradchenko@univ.kiev.ua

1) Работа выполнена при поддержке фонда Александра фон Гумбольдта (грант 1074615). 